\title{
ESTIMATING OPERATIONAL BENEFITS OF AIRCRAFT NAVIGATION AND AIR TRAFFIC CONTROL PROCEDURES USING AN INTEGRATED AVIATION MODELING AND EVALUATION PLATFORM
}

\author{
Ralf H. Mayer \\ Center for Advanced Aviation System Development (CAASD) \\ The MITRE Corporation \\ 7515 Colshire Drive \\ McLean, VA 22102, U.S.A.
}

\begin{abstract}
Complex constraints generally define the performance of air transportation systems. These constraints include aircraft operational characteristics, airline operating procedures, and Air Traffic Control (ATC) requirements. The operational variability that is present in complex air transportation systems and their components typically demands a Monte Carlo approach when modeling system performance metrics. However, the inherent variability is generally not known a priori. This calls for a separate model validation approach that yields estimates of system variability and validates baseline model performance. This paper reports on an integrated aviation modeling platform that was developed for comparing and evaluating proposed aircraft flight operations and ATC procedures. It integrates both an agent-based Monte Carlo modeling environment and a data-driven model validation capability. The capabilities are outlined, the validation approach is described, and examples are presented of performance metrics quantifying operational benefits of air navigation procedures that are currently being implemented at major U.S. airports.
\end{abstract}

\section{BACKGROUND}

The Federal Aviation Administration's (FAA) Operational Evolution Plan (OEP, 2006) for the National Airspace System (NAS) addresses the challenge of managing safe and expeditious flight for an increasing quantity of air traffic. The plan outlines several strategies for improving the efficiency of airport arrival and departure operations and reducing aircraft delays including: (1) airspace redesign and (2) utilization of new aircraft navigation technologies. Airspace redesign can involve changing the shapes and volumes of airspaces assigned to air traffic controllers or the number and location of air routes. The utilization of three-dimensional (3D) navigation capabilities of onboard aircraft Flight Management Systems (FMS) that are available on the majority of today's commercial and corporate aircraft enables automated flight path guidance along predefined routes in terminal airspace in the vicinity of the airport. These routes are often defined by a sequence of Area Navigation (RNAV) waypoints. RNAV waypoints are not necessarily dependent on ground-based navigation aids and enable greater flexibility in the design of diversified navigation routes. Implementation of RNAV procedures is currently underway at many U.S. airports promising more efficient utilization of limited runway capacity and constrained terminal airspace.

\section{INTRODUCTION}

Computer simulations of air traffic are a major source of quantified estimates of system benefits that can arise from implementation of procedural changes. The MITRE Corporation's Center for Advanced Aviation Systems Development (CAASD) was tasked to support the FAA in evaluating potential benefits of proposed operational changes and developed fast-time simulation capabilities. The modeling process and capabilities presented here differ from widely used modeling approaches (TAAM, 2006) in three key areas: it (1) makes data-driven validation of simulation models an integral part of the modeling process, (2) it is agent based, and (3) employs Monte Carlo modeling techniques. The model validation approach recognizes the need to quantify the variability that is intrinsic to flight operations in a complex air transportation system. It involves the comparison of key model performance metrics to performance metrics derived from radar flight track data of actual operations. The agent-based model implementation inherently supports rule-based actions of active model objects and rule execution that is subject to variability. The Monte Carlo modeling approach recognizes this operational variability and yields mean values of performance metrics. The resulting measurement precision is essential to reliably evaluate the effects of proposed operational changes. This is especially important as the impact on relevant aviation system performance metrics is often lim- 
ited and typically results in performance improvements that are on the order of a few percent.

Section 3 of this paper provides an overview of the process for comparing and evaluating aircraft flight operations and ATC procedures as it applies to evaluating RNAV departure operations. Details of the modeling platform are presented in Section 4 and key airport performance metrics of modeled conventional and RNAV departure operations are discussed in Section 5.

\section{EVALUATION PROCESS}

In order to yield operational benefit, it is necessary that proposed aircraft flight procedures or ATC procedures enable operational changes that improve key system performance metrics. The number of aircraft that can depart in a given time interval often serves as a performance metric of an airport. Implementation of RNAV departure procedures can be expected to improve airport departure performance if the procedures enable operational changes that shorten the time between departures, i.e. reduce interdeparture times between aircraft and thus improve departure efficiency.

\subsection{Operational Change}

A key operational change that may result from the design and implementation of RNAV departure procedures is associated with a diversified air route structure that may become available for navigation soon after takeoff. Figure 1 illustrates the operational change associated with RNAV departure procedures that are currently being implemented at major U.S. airports. It compares a typical initial flight pattern of conventional operations that involves a single flow of aircraft from a departure runway to that of RNAV departure operations on two initially diverging route segments. If aircraft that are lining up for departure at a runway can be queued in separate line-up queues (serving initially diverging RNAV departure routes), such separate queues may enable ATC to sequence aircraft for fanned departures, i.e. departure operations that make alternating use of the routes.
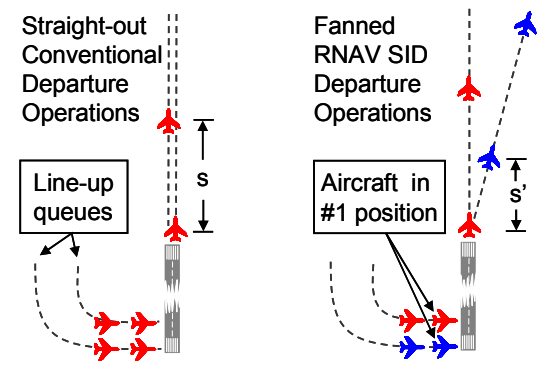

Figure 1: Illustration of Key Operational Changes Associated with Straight-Out Conventional and Fanned RNAV Departure Operations (Notional)
Conducting fanned departure operations generally improves airport departure performance over that associated with straight-out operations and effectively reduces interoperation times between departing aircraft.

\subsection{Benefit Mechanism}

The mechanism that enables operational benefit of fanned departure operations is based on differences in ATC minimum separation standards that apply to straight-out and fanned departure operations [FAA, 2006]. The minimum ATC separation standard that applies most frequently to consecutively departing aircraft operating at large U.S. airports calls for an initial application of 3-nautical mile (NM) spacing between straight-out departures. If the same aircraft can be fanned, a subsequent departure can be authorized to start the takeoff roll if the preceding departure has gained a distance of 6,000 feet and has become airborne. Thus, applicable ATC minimum standards for fanned departure operations generally impose a less stringent constraint and enable ATC to effectively reduce interoperation times between aircraft taking off from a runway. The associated gain in departure efficiency is expected to result in improved departure performance of the airport system.

\subsection{Model Development}

If the presence of significant operational changes and associated benefit mechanisms warrant further model development and evaluation of potential operational benefits, the next step in the modeling process involves identification of key constraints that are relevant in the modeling of operations. Within terminal airspace of many major airports, a large degree of operational independence between departure and arrival operations often suggests tailoring simulation analysis to selectively model either type of operation. For the case of aircraft departure operations, applicable ATC separation standards typically constitute the most significant constraints that limit the departure performance of the airport system. It is imperative that these minimum separation standards are modeled directly. In the modeling approach presented here, the model validation process enables identification and quantification of system-intrinsic variability and any remaining secondary constraints for inclusion in the model such as arrival-departure dependencies that may also impact departure operations.

\subsection{Model Validation}

While applicable ATC separation standards typically constitute the most significant constraints that define the upper bounds of the operational envelope of departure operations, implementation of such standards on a case-by-case basis is subject to significant variability. This variability is due 
to various additional operational constraints including limited voice communications capacity as well as air crew procedural requirements resulting in actuation delays or controller/flight crew performance, style, and workload, and will typically vary from one departure to the next. The objective of the model validation process is to (1) quantify the system-intrinsic variability that exists in actual operations and (2) account for other dependencies that are not otherwise captured by the direct modeling of the most significant constraints. For the modeling of aircraft navigation and ATC procedures at large U.S. airports, Terminal Radar Approach Control surveillance data of actual flight operations are often available for analysis to determine the operational characteristics required for validation of aviation simulation models. This typically involves selecting a metric that is best suited for sensitively measuring the impact of the operational changes under evaluation. When modeling the impact of operational changes associated with the implementation of RNAV departure procedures, the distribution of observed inter-departure times was found to be a sufficiently sensitive metric for the purpose of validating model behavior. The model validation process that confirms good agreement between the model of departure operations and observed operations suggests that significant operational requirements constraining actual operations are sufficiently accounted for in the model. The performance of the validated model then provides a performance baseline for comparing operational alternatives and estimating their potential operational benefits.

\subsection{Benefit Estimation}

Potential benefits associated with RNAV-enabled fanned departure operations arise from application of ATC minimum separation standards that impose a less stringent constraint on airport departure performance than those applicable to conventional straight-out departure operations. It is important to note that full realization of these benefits is contingent upon ATC's ability to effectively sequence aircraft for fanned departure operations. Whenever sufficient departure demand exists and fanned departure operations can be conducted, they can be characterized as shortestprocess-next (SPN) operations and may represent a deviation from the first-come-first-served (FCFS) operational principle that more generally characterizes current ATC sequencing processes. Benefits arising from application of effectively reduced inter-departure times and improved ATC departure separation efficiency can be expected to result in increased departure capacity. For given departure demand, a gain in departure capacity in turn enables reductions in departure delay. The modeling platform developed by The MITRE Corporation that enables flexible and detailed evaluations of delay and capacity benefits is described in the following section.

\section{MODELING PLATFORM}

The modeling platform was constructed using the programming language Simulation Language with eXtensibility (Henriksen, 1998). SLX is a highly flexible objectbased general simulation programming language. MITRE's Integrated Terminal Research, Analysis and Evaluation Capabilities (iTRAEC) presented here comprises two complementary capabilities.

The first capability is a flexible discrete-event aviation modeling tool set that comprises object classes whose actions are designed to mirror flight operations as well as ATC control activities. This capability serves as Benefit Analysis (iTRAEC-B) simulation platform designed for model evaluation of proposed flight navigation and ATC decision-making processes that are subject to operational and procedural constraints. iTRAEC-B is supported by SLX tools for generating stochastic variations of modeling parameters and employs a fast 4D flight trajectory generation capability that supports Monte Carlo techniques involving the modeling of large numbers of flight operations (Mayer, 2003).

The second capability serves as Operational Analysis (iTRAEC-O) tool for the evaluation of radar surveillance data of actual flight operations. iTRAEC-O permits determining key operational performance characteristics of flight operations and ATC control activities from operational data and enables the validation of iTRAEC-B models of baseline operations. A key feature of the iTRAEC platform is that both iTRAEC-B and iTRAEC-O share iTRAEC procedures for collecting and analyzing performance metrics as well as Proof Animation-based visualization and animation capabilities (Wolverine, 2004).

\subsection{Benefit Analysis Capability (iTRAEC-B)}

iTRAEC-B's aircraft flight performance is based on Eurocontrol's Total Energy Model and its Base of Aircraft Data (BADA). BADA provides performance parameters for 84 aircraft types commonly used in commercial air carrier operations (Eurocontrol, 2004). The data base also supports 180 additional aircraft types by assigning each additional type to one of the 84 directly modeled types with similar performance characteristics. Aviation infrastructure information is represented in iTRAEC-B by Enhanced Traffic Management System (ETMS) data. The FAA uses ETMS data to characterize the locations of runways, airways, and other aviation infrastructure for managing the flow of air traffic within the NAS. The ETMS system also serves as source for air traffic information that is key model input when simulating aviation system performance. Depending upon the modeling application, iTRAEC-B simulations that evaluate airport capacity benefits may employ ETMS-based probability distributions that specify the likelihood of a specific aircraft type to operate at the 
airport under investigation (Mayer, 2005). Or, if iTRAEC$B$ is adapted to evaluate airport delay benefits, ETMSbased traffic information regarding each flight operating at the airport of interest on a specific day are accepted as input to iTRAEC-B on a flight-by-flight basis. Each flight's traffic information typically comprises FAA flight planning information including the flight's call sign, aircraft type, navigational equipment, planned push-back time, initial cruising altitude, and route of flight.

\subsubsection{Aircraft Flight Agent}

For each flight, iTRAEC-B instantiates an object of class Flight using the flight planning information for initialization. The actions of each Flight object successively define and schedule the events that are significant in characterizing the Flight's various states, or phases, and its 4D trajectory along the planned route. This flight phase-based approach to simulating flight trajectories facilitates the modeling of state-based interactions between flight operations and ATC control activities. For example, at the time a flight is scheduled to push back from its gate, the state of the flight is modeled to change from "SCHEDULED" to "READY-TO-TAXI". This is conveniently captured in SLX through the use of control variables. For example, a change of an SLX control boolean in the Flight object may be used to trigger state-based execution of actions of Controller objects.

\subsubsection{Ground Controller Agent}

At the time an aircraft is scheduled to push back from the gate, a flight is put under the control of iTRAEC-B's Ground Control agent. Actions of the Ground Control agent include assigning a departure runway to a flight.

The Ground Control agent determines which runways are suitable based on the flight's route or aircraft type. If only one runway is suitable, that runway is assigned as departure runway. If more than one runway is available, the Ground Control agent may implement dynamic runway usage strategies and, for example, assign the runway whose departure queue length is the shortest at that time.

The Ground Control agent then assigns a departure procedure to a departing flight. This assignment is generally based upon the cardinal flight direction of the departure, the route specified in the flight's flight plan, and aircraft type. The departure procedure provides routing information for navigation within the terminal airspace. The Ground Control agent also schedules a time that represents the time required for the aircraft to taxi to the hold line of the assigned departure runway or, more often, the time to join a line-up queue that has formed there for departure (see Figure 1). iTRAEC-B's unconstrained model of ground operations ensures highly efficient handling of ground movements from the time flights are scheduled to push back from their gates and until aircraft become airborne. This ground model meets a key design requirement by enabling efficient operations over a large range of operations activity levels, i.e. when departure demand is increased well above the baseline level. It allows to quantify benefits of operational changes that solely result from proposed airspace design, air navigation, or ATC procedure changes and that are not impacted by constrained ground movement resources.

\subsubsection{Local Controller Agent}

Upon expiration of its taxi time, control of a flight is assumed by iTRAEC-B's Local Control agent. The Local Control agent adds the flight to the appropriate line-up queue based on its assigned departure runway and flight route (see Figure 1). Key actions of the Local Control agent are associated with the sequencing of aircraft for departure and separating departing aircraft from aircraft that departed previously.

When evaluating potential benefits of RNAV departure procedure implementations, it is important to note that the associated departure efficiency benefits rely on ATC controller actions that leverage opportunities to sequence aircraft for fanned departures, i.e. departure operations that make alternating use of initially diverging routes (see Section 3.2). A model of associated ATC decision making processes is required to explore the range of sequencing options and to evaluate the bounds of the resulting model performance. The sequencing algorithms that govern the actions of iTRAEC-B's Local Control agent are designed to evaluate the spectrum of possible ATC control strategies.

The first strategy assures that equity is given to departures. In this case, the Local Control agent strictly enforces ATC service on a $\boldsymbol{F C F S}$ basis.

The second strategy evaluates separation requirements of all aircraft that occupy \#1 positions in their respective line-up queues and gives precedence to $\boldsymbol{S P N}$ aircraft, i.e. aircraft that can be released for earliest departure. This strategy optimizes an airport's departure performance. When executed, iTRAEC-B's Local Control agent monitors the position of each flight within its departure line-up queue. Every time a flight advances to the $\# 1$ position within its departure line-up queue, i.e. the flight reaches the hold-short line at a runway, the Local Control agent sequences all flights in \#1 positions for departure on that runway. The separation algorithms employ flight trajectory information and applicable ATC separation minima. Flight trajectories are calculated based on the aircraft type and assigned departure procedure (Mayer, 2003). For a given flight that departed previously, each possible trailing aircraft currently lined up in \#1 position is evaluated. The Local Control agent assigns as departure time the earliest possible time an aircraft can depart that meets ATC separa- 
tion requirements. If no further sequencing action is required that revises a flight's departure time, a flight is modeled to begin its takeoff roll when iTRAEC-B's simulation time has advanced to a flight's last assigned departure time.

In order to account for variability present in actual operations - due to actuation delays as well as differences in controller/flight crew performance, style, and workload (see Section 3.4) - iTRAEC-B's modeled departure times are subjected to stochastic variability. iTRAEC Operational Analysis capability is used to determine the amount of stochastic variance observed in radar flight track data of actual operations as part of the model validation process outlined in the following section.

\subsection{Operational Analysis Capability (iTRAEC-O)}

iTRAEC aircraft Flight agent that serves to model iTRAEC-B 4D flight trajectories is replaced in iTRAEC-O by data parsing and processing capabilities. Both, iTRAEC-O and iTRAEC-B share common iTRAEC procedures for collecting and analyzing performance metrics as well as Proof Animation-based visualization and animation capabilities. While iTRAEC-B's performance metrics are a result of modeled air traffic based on flight schedule information input as well as the interactions between active Flight and Controller agents, iTRAEC-O enables flight and airport performance evaluations that are based on radar surveillance data of actual flight operations. Comparisons of iTRAEC-B and iTRAEC-O performance metrics allow to identify and quantify the system-intrinsic variability that exists in actual operations and other operational dependencies for inclusion in iTRAEC-B models of baseline and proposed operations. The model validation process that is found to result in good agreement between iTRAEC-B performance metrics of the baseline model and performance metrics derived from iTRAEC-O analyses of radar surveillance data of actual flight operations suggests that all significant operational constraints characterizing actual operations are sufficiently accounted for in the model.

\subsubsection{Model Validation}

The efficiency of departure operations is characterized here by the distribution of separation times observed between consecutive departures. It is important to note that this distribution mainly reflects two possible operational scenarios. In the first scenario, continued departure demand exists and observed separations are largely a result of ATC's continuous implementation of minimum separation standards. In the second scenario, a lack of continuous departure demand yields separations that largely reflect the temporal distribution of departure demand. Figure 2 illustrates typical distributions of inter-departure times deduced from radar data recorded during six days of departure op- erations at a large U.S. airport. Each observed distribution comprises nearly 1000 separation measurements of actual departure operations. The modes of the observed distributions are seen to represent the most frequently applied separations with values of about 60 to 70 seconds. These modes mainly reflect those operational scenarios in which uninterrupted departure demand resulted in ATC's continuous implementation of minimum separation standards that call for initial application of 3-NM spacing between consecutive straight-out departures. The modes largely characterize the variability resulting from varying flight crew actuation delays as well as differences in controller performance, style, and workload. They allow the deduction of a mean value of observed actuation delays and associated variance. The mean and variance values can be viewed to characterize average flight crew and controller performance variability and to validate iTRAEC-B model performance as shown in Figure 2. The figure indicates generally good agreement between inter-departure time distributions observed in actual operations and the distribution deduced from iTRAEC-B model output validating the iTRAEC-B performance of the baseline model of straightout conventional departure operations (see Figure 1).

The operational variability parameters that were found to validate the model of baseline operations are assumed to also apply to alternative models of proposed operations. This approach is based on the assumption that operational constraints such as flight crew actuation delays as well as controller performance, style, and workload remain unchanged when alternative operations are in effect. Benefit evaluations of alternative operations then generally involve the comparison of baseline and alternative model performances as described in the following section.

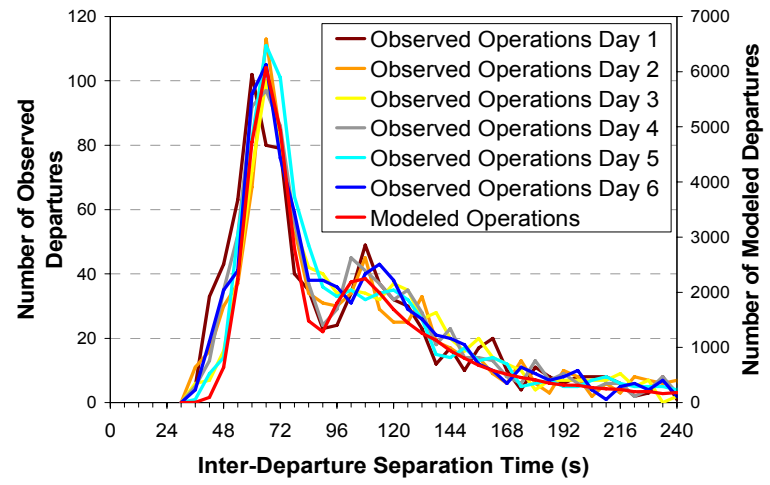

Figure 2: Inter-Departure Separation Time Distributions of Observed and Modeled Departure Operations

\section{BENEFIT EVALUATION}

When evaluating potential benefits of RNAV departure procedures, the alternative model of proposed operations differs from the model of baseline operations insofar as iTRAEC-B's Local Control agent implements procedural 
constraints that are adapted to reflect operational changes associated with proposed procedures. These constraints may involve leveraging opportunities to sequence aircraft for fanned departures. They require application of appropriate separations between all combinations of departures that make sequential or alternating use of straight-out or initially diverging RNAV departure routes (see Figure 1). The following sections outline iTRAEC-B model input, discuss modeling assumptions, and present key performance metrics.

\subsection{Model Input and Assumptions}

Evaluating airport delay benefits of RNAV departure procedures generally requires an input traffic file that characterizes the departure demand distribution that existed at the airport of interest on a selected day that meets average or above-average traffic volume requirements. The adopted baseline traffic file may also serve to generate future demand scenarios in which departure demand is increased according to growth rates that are typically based on FAA traffic volume growth forecasts for the airport.

The baseline traffic file represents an aircraft fleet mix comprising numerous aircraft types and navigational capabilities currently operating at the airport under investigation. At most U.S. airports offering current types of RNAV departure procedures, the navigational equipment onboard 80 to 90 percent of commercial and corporate aircraft authorizes operators to accept RNAV departure clearances. These RNAV participation rates and resulting operational benefits are expected to increase as more aircraft are being equipped with advanced navigation capabilities.

The ATC strategy that is applied when sequencing RNAV departure operations for fanned departures impacts the degree to which departure efficiency benefit opportunities are realized (see Section 4.1.3). The benefit results reported in this study are based on the assumption of an ATC sequence optimization rate of 80 percent. In other words, ATC operational practice is assumed to enable conducting $S P N$ operations in 80 percent of the cases in which a need for a sequencing decision arises while FCFS operations are conducted in 20 percent of these cases.

Airport departure delay not only is a function of the total number of departures but also of how scheduled departure demand is distributed over the course of a day. Delay can also be expected to vary as actual aircraft push-back times of scheduled air carrier operations vary from one day to the next. In order to extend the validity of iTRAEC-B modeling results beyond a selected single traffic day, stochastic variations are introduced to the gate pushback times of flights and multiple replicates of simulation runs are performed. The gate push-back time variance represents the main stochastic element in iTRAEC-B modeling of departure operations. A normal distribution with a standard deviation of 180 seconds was selected to represent the vari- ance characterizing actual push-back and taxi operations. Introduction of this amount of variance was chosen to remove some of the day-specific nature of a particular traffic input scenario used in iTRAEC-B departure delay modeling.

The modeling platform presented here defines a flight's departure delay as any time it spends after joining a line-up queue at the runway up to the moment the flight commences the takeoff roll on the departure runway. In other words, aircraft departure delay is defined as difference between the actual departure time of a flight and the time it completes taxiing and joins a line-up queue at a runway. The term runway delay could also be used to describe this delay metric. Extending this definition to include all daily departure operations represented in a traffic file, airport departure delay is defined here as the sum of all daily aircraft departure delays.

\subsection{Model Convergence}

Average airport departure delay serves as key metric in estimating delay benefits of proposed operational changes. Airport departure delay averages that are based on 50 Monte Carlo replicates of simulation runs comprising approximately 50,000 simulated operations were found to display adequate convergence. Figure 3 illustrates the observed convergence of the average airport delay metric of modeled baseline departure operations.

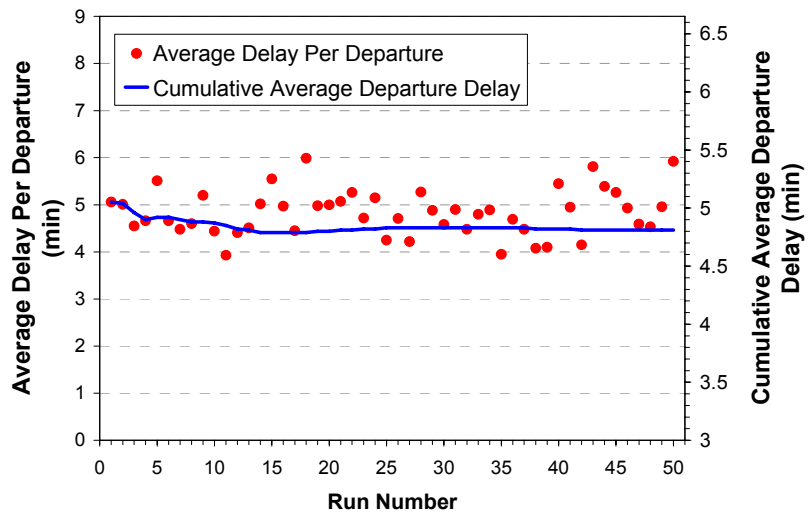

Figure 3: Convergence of the Modeled Average Airport Departure Delay Metric

\subsection{Procedure Benefits}

Examples of airport delay benefit metrics associated with implementation of RNAV departure procedures are presented in this section. These examples illustrate iTRAEC$B$ model evaluation results of operational benefits of fanned departure operations that can be viewed as typical for operations conducted at large U.S. airports. 


\subsubsection{Departure Efficiency Benefits}

The departure efficiency metric (see Section 4.2.1) evaluates changes in effectively applied aircraft separations between consecutive departures from a runway. Modeled inter-departure time distributions of straight-out and fanned RNAV departure operations are presented in Figure 4. The distribution of inter-departure separation times resulting from fanned operations illustrates the impact of operational changes associated with implementation of RNAV departure procedures (see Sections 3.1 and 3.2). The pronounced mode of the distribution that is visible for conventional operations at about 60 to 70 seconds of interdeparture time is essentially split allowing a sizable number of fanned departures to be spaced more closely, i.e. by about 40 to 50 seconds.

The distribution associated with RNAV departure operations also features increased numbers of departures that are spaced about 100-110 seconds apart. This operational change reflects the impact of additionally required spacing rules iTRAEC-B's Local Control agent implemented when separating consecutive RNAV and non-RNAV departures. This example reflects specific operational needs that may require ATC to occasionally apply additional spacing to meet local operational constraints associated with a specific design and implementation of RNAV routes. The examples presented in the following sections are based on the modeled inter-departure time distributions presented in Figure 4.

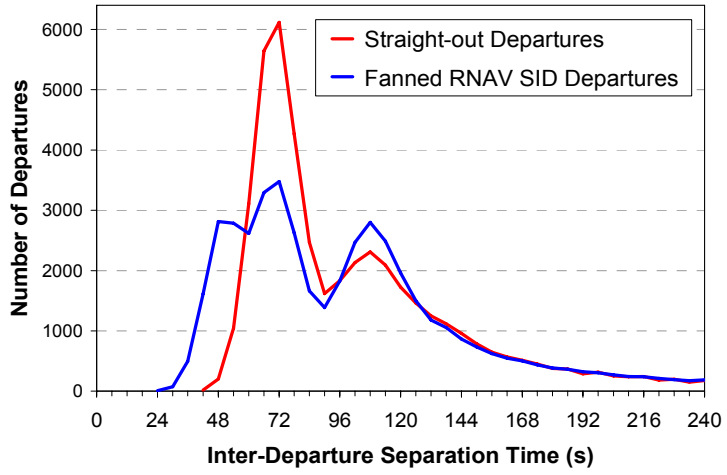

Figure 4: Comparison of Modeled Inter-Departure Time Distributions

\subsubsection{Delay Reduction Benefits}

Delay distributions often serve as metric for evaluation of delay aspects of operational changes. Delay distributions illustrate how departure delay is distributed over measured departure delay values. Modeled delay distributions of straight-out and fanned RNAV departure operations are presented in Figure 5. The distribution of departure delays resulting from fanned departure operations illustrates the impact of operational changes associated with implementation of RNAV departure procedures. While slightly more

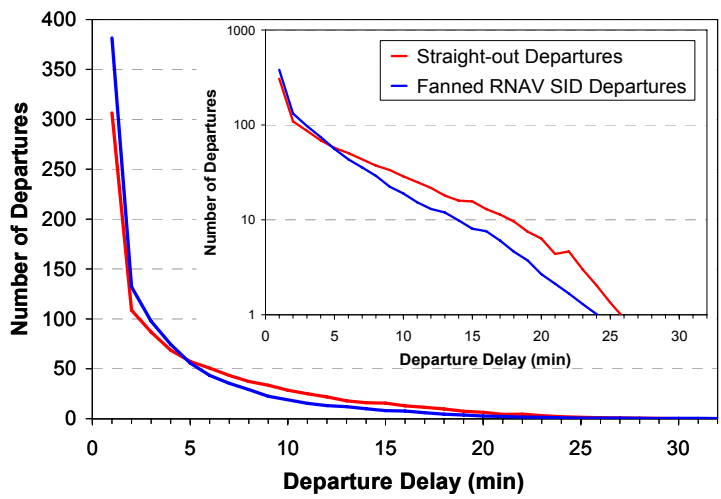

Figure 5: Comparison of Modeled Departure Delay Distributions

than 300 departures are seen to accrue up to 1 minute of delay in straight-out operations, this number is seen to increase to about 380 in fanned RNAV departure operations. Conversely, RNAV departure operations are seen to involve fewer departures that accrue larger values of departure delay starting at about 5 minutes of delay.

Average delay per departure is a metric that is frequently used to characterize the departure performance of an airport. Modeled average departure delays associated with straight-out and fanned RNAV departure operations are presented in Figure 6 . The average delay values resulting from fanned departure operations illustrate the impact of operational changes associated with implementation of RNAV departure procedures as departure demand is assumed to increase as shown in the figure. These examples illustrate how fanned departure operations not only result in reduced average departure delays but also indicate a lower rate of increase in departure delay as departure demand increases. The results reflect a key characteristic of the underlying benefit mechanism that relies on the existence and incrementally leverages increasing departure demand that provides ATC with more opportunities to sequence aircraft for fanned departures.

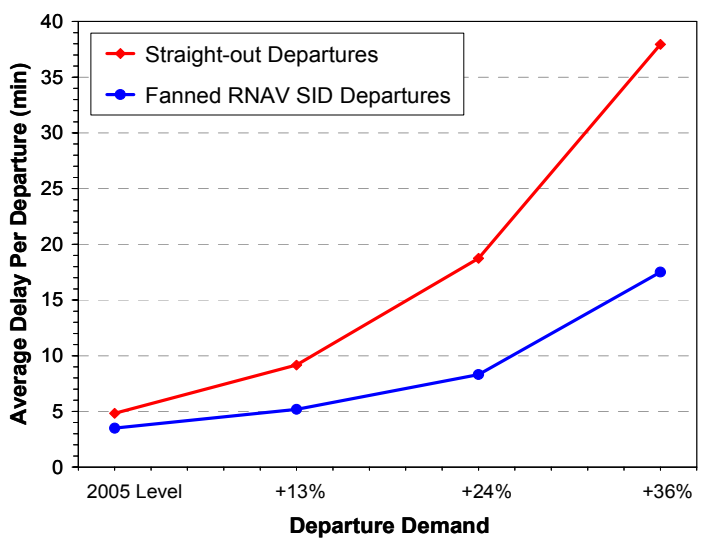

Figure 6: Comparison of Modeled Average Departure Delays 


\subsubsection{Departure Throughput and Capacity Benefits}

The departure throughput metric is typically based on actual departure demand as represented in a traffic file that is input to a model (see Section 5.1). It evaluates changes in the number of operations that are conducted within a given time interval. Modeled average throughput histograms associated with straight-out and fanned departure operations are presented in Figure 7. The histogram resulting from fanned departure operations illustrates generally increased departure throughput during 15-minute time intervals with peak departure demand.

A capacity metric is used more commonly to estimate the average number of operations an airport can conduct in a given time interval and independent of the temporal distribution of demand. Thus, capacity modeling generally evaluates a scenario in which departure demand is continuous. It provides an estimate of maximum sustainable throughput, on a long-term basis, given sustained demand (Lisker-Melmar, 2000). Adopting iTRAEC-B to provide continuous departure demand, the increases in departure capacity associated with fanned departure operations characterize the impact of operational changes associated with implementation of RNAV departure procedures. Capacity benefit results indicate that capacity increases of about 10 additional departure operations per hour and runway are possible at large U.S. airports.

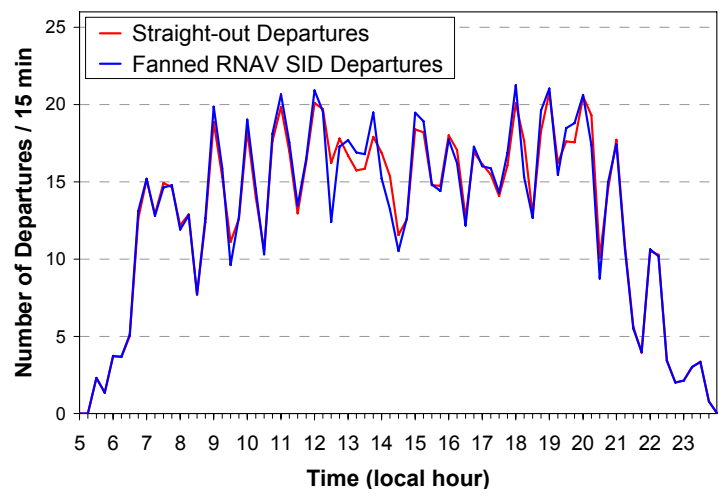

Figure 7: Comparison of Modeled Average Throughput

\section{CONCLUSIONS}

An integrated aviation modeling platform was developed for comparing and evaluating proposed aircraft flight operations and ATC procedures. It comprises both an agentbased Monte Carlo modeling environment and a datadriven model validation capability. It enables a comprehensive modeling approach that includes the quantification of operational variability present in complex air transportation systems. The modeling platform was applied to evaluate potential benefits associated with the implementation of RNAV departure procedures currently underway at large U.S. airports. Examples of various airport performance metrics indicate significant operational benefits of fanned departure operations and promise more efficient utilization of limited runway capacity and constrained terminal airspace.

\section{ACKNOWLEDGEMENTS}

The author would like to thank Mr. Thang D. Phung who prepared the traffic files and Mr. Tyler M. Smith for his help with obtaining the radar track data that enabled the validation of the model.

The contents of this material reflect the views of the author and/or the Director of the Center for Advanced Aviation System Development. Neither the Federal Aviation Administration nor the Department of Transportation makes any warranty or guarantee, or promise, expressed or implied, concerning the content or accuracy of the views expressed herein.

\section{REFERENCES}

Eurocontrol. 2004. Base of Aircraft Data (BADA 3.5), The EUROCONTROL Experimental Centre, Brétigny, France.

FAA. 2006. Federal Aviation Administration Order 7110.65R, Air Traffic Control, Washington, D.C.

Henriksen, James O. 1998. Stretching the boundaries of simulation software. In Proceedings of the $1998 \mathrm{Win}$ ter Simulation Conference, ed. Medeiros, D.J., E. Watson, M.S. Manivannan, and J. Carson, 227-234. Piscataway, New Jersey: Institute of Electrical and Electronics Engineers.

Lisker-Melmar, Dr. Bernardo, et al. 2000. Future Airport Development for Mexico City, Studies of Technical Feasibility, MTR 00W0000090, The MITRE Corporation, McLean, VA.

Mayer, Ralf H., 2003, A Flight Trajectory Model for a PCBased Airspace Analysis Tool, In Conference Proceedings of the AIAA Modeling and Simulation Technologies Conference and Exhibit, Austin, TX.

Mayer, Ralf H., and Howard Swancy. 2005. Analysis of Potential Benefits of arrival-departure procedures proposed for ORD, In Conference Proceedings of the $24^{\text {th }}$ Digital Avionics Systems Conference, Washington, DC.

Mayer, Ralf H. 2005. Analysis of Potential Benefits of Wind Dependent Parallel Arrival Operations, In Conference Proceedings of the $24^{\text {th }}$ Digital Avionics Systems Conference, Washington, DC.

OEP. 2006. Federal Aviation Administration, Federal Aviation Administration National Airspace System Operational Evolution Plan, Version 7.1, Washington, D.C. 
TAAM. 2006. Total Airport and Airspace Modeller, Preston Aviation Solutions, Pty Ltd, Richmond, Australia

Wolverine. 2006. Wolverine Software Corporation, Alexandria, VA

\section{BIOGRAPHY}

RALF H. MAYER is a Lead Simulation Modeling Engineer at The MITRE Corporation's Center for Advanced Aviation System Development (CAASD). He received a Ph.D. degree from Purdue University, has over 10 years of research experience in aviation, experimental nuclear and biomedical physics, and is an author of over 30 scientific journal publications. Dr. Mayer holds commercial pilot and flight instructor certificates and served on the staff of Purdue University's Aviation Technology Department before joining CAASD in 2001. He has been working on capacity/delay/fuel burn benefit evaluations of airspace and procedure designs. His research interests are fast-time, agent-based simulation and Monte-Carlo modeling. He can be contacted by e-mail at <rmayer@mitre.org >. 